\title{
Value-modulated oculomotor capture by task-irrelevant stimuli is a consequence of early competition on the saccade map
}

\author{
Daniel Pearson ${ }^{1} \cdot$ Raphaella Osborn $^{1} \cdot$ Thomas J. Whitford $^{1} \cdot$ Michel Failing $^{2}$ • \\ Jan Theeuwes $^{2} \cdot$ Mike E. Le Pelley $^{1}$
}

Published online: 16 May 2016

(C) The Psychonomic Society, Inc. 2016

\begin{abstract}
Recent research has shown that reward learning can modulate oculomotor and attentional capture by physically salient and task-irrelevant distractor stimuli, even when directing gaze to those stimuli is directly counterproductive to receiving reward. This value-modulated oculomotor capture effect may reflect biased competition in the oculomotor system, such that the relationship between a stimulus feature and reward enhances that feature's representation on an internal priority map. However, it is also possible that this effect is a result of reward reducing the threshold for a saccade to be made to salient items. Here, we demonstrate value-modulated oculomotor capture when two reward-associated distractor stimuli are presented simultaneously in the same search display. The influence of reward on oculomotor capture is found to be most prominent at the shortest saccade latencies. We conclude that the value-modulated oculomotor capture effect is a consequence of biased competition on the saccade priority map and cannot be explained by a general reduction in saccadic threshold.
\end{abstract}

Keywords Eye movements and visual attention - Attentional capture $\cdot$ Attention in learning

Traditionally, attention has been argued to be subject to two different types of control: one that is volitional and goal directed (top-down control) and another that is automatic and

Daniel Pearson

d.pearson@unsw.edu.au

1 School of Psychology, UNSW Australia, Sydney, NSW 2052, Australia

2 Department of Experimental and Applied Psychology, Vrije Universiteit Amsterdam, Amsterdam, The Netherlands stimulus driven (bottom-up control) (Yantis, 2000). Recently, however, a case has been made for a third category of influences on attentional selection that is neither goal directed nor stimulus driven. Specifically, it has been suggested that our attention is influenced by what we have learned about how stimuli relate to other events in the environment (Anderson, 2013; Awh, Belopolsky, \& Theeuwes, 2012; Chelazzi, Perlato, Santandrea, \& Della Libera, 2013; Le Pelley, Mitchell, Beesley, George, \& Wills, 2016; Le Pelley, Pearson, Griffiths, \& Beesley, 2015; Mitchell \& Le Pelley, 2010).

The suggestion that attention and learning might interact has a long history in the conditioning literature (Kamin, 1968; Mackintosh, 1975; Trabasso \& Bower, 1968; for a review, see Le Pelley, 2004). However, recent work-much of it from the lab of Steven Yantis - has reinvigorated this idea by demonstrating conclusively that learning about the relationships between stimuli and rewards influences the likelihood that those stimuli will automatically capture attention (e.g., Anderson, Laurent, \& Yantis, 2011a, 2011b; Anderson \& Yantis, 2012; Della Libera \& Chelazzi, 2009; Failing \& Theeuwes, 2014; Hickey, Chelazzi, \& Theeuwes, 2010, 2011; Le Pelley et al., 2015; Pearson, Donkin, Tran, Most, \& Le Pelley, 2015; Rutherford, O'Brien, \& Raymond, 2010; Theeuwes \& Belopolsky, 2012; for a systematic review, see Le Pelley et al., 2016). Specifically, these studies demonstrate that stimuli associated with high-value rewards are more likely to capture attention than equally salient stimuli that are associated with low rewards (or no reward). For example, Anderson et al. (2011b) gave participants extensive pretraining on a visual search task in which red and green circles defined the targets and also signalled the reward magnitude that would be earned for a rapid response to those targets. If the target circle was rendered in (say) red, participants would receive a relatively large reward $(5 \phi)$ for a rapid correct keypress response, 
whereas if the target was rendered in green, the participant would receive a relatively small reward $(1 \phi)$ for the same response. Thus, in the example given here, red acted as the high-value colour and green acted as the low-value colour (these colour-reward contingencies were reversed for half of the participants). In a subsequent test phase, the target was defined by shape (e.g., a diamond in an array of circles), and participants no longer received rewards for their responses. On $50 \%$ of trials in the test phase, one of the nontarget shapes (termed the distractor) was rendered in either the high-value colour or the low-value colour. The critical finding was that the distractor rendered in the high-value colour was more likely to capture attention than a distractor that had never been rewarded. This occurred despite the fact that participants were explicitly informed that colour was no longer relevant to the task during the test phase and that all shapes in the search display were uniquely coloured, such that the rewardassociated distractor was not physically salient. This was taken as evidence to suggest that reward learning influences attentional capture separately from stimulus-driven and goaldirected processes.

Similar effects have been reported in studies of eye movements. For example, Theeuwes and Belopolsky (2012) used a gaze-contingent visual search task that was conceptually similar to Anderson et al. (2011b), in which participants were required to make a saccade to a target stimulus (a horizontal or vertical bar) presented in an array of other shapes during an initial pretraining phase. If the participant made an accurate and fast saccade to one of these target stimuli, they would receive a high-value reward, whereas those to the other target stimulus were followed by relatively low-value reward. In a subsequent test phase in which participants were to make a saccade to a colour-defined target, eye gaze was more likely to be captured by a distractor that had the shape that was previously paired with high-value rewards, compared to the shape that had been paired with low-value rewards.

A common feature of both of these studies is that the stimuli that captured attention during the critical test phase were task relevant during the initial training phase. That is, participants needed to quickly orient to the target stimulus in order to earn the reward for that trial. This raises the possibility that the attentional capture by these stimuli in the test phase (where attending to the stimulus was no longer relevant to the participant's goals) was simply a carryover of the attentional and/or oculomotor orienting response that was initially trained. It is well known from the reward-learning literature that following an action with reward increases the likelihood that the action will occur in the future (Thorndike's law of effect; Thorndike, 1911). Perhaps, then, it is not surprising that a rapid orienting response that was followed by large reward in the training phase continues to occur in a subsequent test phase, even when the response is no longer relevant to the task goals. That is, it is possible that the attentional and oculomotor orienting observed in these studies simply reflects a learned (conditioned) response that is automatically reenacted whenever the relevant conditioned stimulus appears (see also Beesley, Pearson, \& Le Pelley, 2015).

However, a recent series of studies by Le Pelley et al. (2015; see also Pearson et al., 2015) has demonstrated that reward value modulates attentional and oculomotor capture, even when attending to the reward-predicting stimulus has never been task relevant. For example, in Le Pelley et al.'s Experiment 3, participants engaged in a gaze-contingent visual search task in which they were required to make a rapid saccade towards a shape singleton target (a diamond in an array of circles). On most trials, a colour-singleton distractor circle was present in the display, rendered in either red or blue (all other shapes were grey). Critically, the colour of this distractor signalled the size of the reward available on that trial. For example, a red distractor might signal that a rapid saccade to the diamond target would earn a large reward (10 $\notin$ ), whereas a blue distractor signalled that a rapid saccade to the target would earn a small reward (1ф). Note that while the coloured distractor signalled reward magnitude, it was not the target that participants were required to respond to in order to obtain that reward; in this sense, it was task-irrelevant. Indeed the task was arranged such that if ever any gaze was recorded on or near the distractor circle prior to looking at the target, the reward that would otherwise have been delivered on that trial was cancelled: These trials were called omission trials. Therefore, throughout the experiment, participants were never rewarded for shifting their gaze to the coloursingleton distractor. In fact, making an eye movement to the distractor was directly counterproductive because it cancelled the reward and hence resulted in a lower payoff for participants. Nevertheless, participants triggered more omission trials when the high-value distractor was present in the display than when the low-value distractor was present. That is, participants were more likely to make eye movements towards the high-value distractor than the low-value distractor, even though this led to the cancellation of more high-value rewards than low-value rewards. This suggests that people develop an attentional and oculomotor bias towards stimulus features that signal high reward, even when orienting to those features has never been relevant to obtaining the reward. We have termed this effect value-modulated oculomotor capture (VMOC; Pearson et al, 2015).

Given that the distractor stimulus in the search display of Le Pelley et al.'s (2015) procedure was physically salient (since it was a colour singleton), we might expect it to capture attention and eye movements on the basis of this physical salience in a stimulus-driven fashion (Theeuwes, 1992, 1994; Theeuwes, Kramer, Hahn, Irwin, \& Zelinsky, 1999). However, the results of this study show that the physical characteristics of the stimuli cannot be the only determinants of attentional capture, because the salience of the high- and lowvalue distractors was matched across participants by counterbalancing. The implication is that the likelihood of 
capture is also influenced by learning about the size of the reward signalled by the distractor, independently of its physical salience (hence our description of value-modulated, rather than value-driven, capture). Furthermore, as the VMOC effect is directly counterproductive to the participant's goal of maximizing reward, it seems that the processes responsible are automatic rather than being under the control of a top-down selection strategy (see also Pearson et al., 2015). In line with this idea, it has been suggested that pairing a particular stimulus feature (e.g., red colour) with reward increases the strength of that feature's representation on attentional (Awh et al., 2012) and saccadic (Belopolsky, 2015; Theeuwes \& Belopolsky, 2012) priority maps.

Several models of oculomotor selection have suggested that the relative priorities of all stimuli in the visual field are represented on a topographical saccade map (e.g., Itti \& Koch, 2001; Li, 2002; Wolfe, Cave, \& Franzel, 1989), which is generally argued to be located in the superior colliculus (e.g., Godijn \& Theeuwes, 2002; Trappenberg, Dorris, Munoz, \& Klein, 2001). The activity on this map determines which stimuli are selected by the visual system, with eye movements and attention being directed to the object that generates the largest peak of activity. In many of these models, the strength of a stimulus' representation on the map is purely a consequence of its stimulus-driven salience, such that the more physically distinct a stimulus is from its surroundings, the greater its associated activity (e.g., Itti \& Koch, 2001; Li, 2002). However, several findings have suggested that the priority map also incorporates goal-directed inputs to the oculomotor system (albeit more slowly) through a process of competitive integration (Godijn \& Theeuwes, 2002; Meeter, Van der Stigchel, \& Theeuwes, 2010; Trappenberg et al., 2001). According to this competitive integration model (Godijn \& Theeuwes, 2002), when a search display containing a target and a colour-singleton distractor is presented to an observer, two peaks of activity are produced on the saccade map. Activity at each of these locations is assumed to spread to nearby locations, while inhibiting more distant activity. Once the activity at one point on the map passes a certain threshold, a saccade is made towards that location. This model can be usefully applied to a number of phenomena that arise from placing goaldirected and stimulus-driven activity in competition with one another. For example, when a salient onset distractor is presented in a relatively distant location from a target, the latency of correct saccades to the target is increased (remote distractor effect; Godijn \& Theeuwes, 2002). This suggests that the stimulusdriven saccadic activity associated with the salient distractor inhibits the goal-directed saccadic activity associated with the target, such that it takes longer for the target program to reach the threshold for a saccade to be made.

According to the competitive integration account, as it was originally formulated, early saccades are driven purely by the physical salience of a stimulus, whereas the effect of goaldirected, top-down influences can be observed on slower saccades. However, as noted earlier, recent studies have identified an influence of reward prediction as a distinct influence on attention. This raises the question of how reward exerts its effect on the saccade map in the process of competitive integration. In particular, if early competition in the saccade map is purely driven by the physical salience of a stimulus, with other nonsensory signals being integrated at a later stage (as is commonly assumed; e.g., Donk \& van Zoest, 2008; Godijn \& Theeuwes, 2002; Ludwig \& Gilchrist, 2002, 2003a, 2003b; Mulckhuyse, van Zoest, \& Theeuwes, 2008; van Zoest, Donk, $\&$ Theeuwes, 2004), then we should expect to see no influence of reward on early saccades; instead, as in the case of goaldirected influences, an effect of reward may emerge only for slower eye movements. In contrast, if reward exerts its influence early on in visual processing, either by enhancing the bottom-up salience signal of the reward-associated stimulus in low-level cortex (Hickey et al., 2010) or through independent inputs to the saccade map that engage in direct competition with the bottom-up salience signal (Belopolsky, 2015), we would expect the effect of reward to be largest when saccade latency is short, because the slow, top-down target selection process has yet to be engaged. The experiments described in the current article provide a direct test of this idea.

As noted above, previous studies (Le Pelley et al., 2015; Pearson et al., 2015) have demonstrated that reward exerts an influence on oculomotor capture by task-irrelevant stimuli that is independent of physical salience in speeded search tasks, which might suggest that reward influences early competition on the saccade map. However, in these studies, the critical reward-predicting stimulus was a physically salient colour singleton. As a result, it is possible that the observed influence of reward on eye movements was not a consequence of reward enhancing activity at the locations of reward-related features on the saccade map at all. Instead, it may be the case that the presence of a stimulus feature associated with a highvalue reward simply lowers the threshold for a bottom-up saccade to be made to any salient stimulus (Theeuwes \& Belopolsky, 2012; see also Anderson, Lauren, \& Yantis, 2013). Thus, oculomotor capture by the salient rewardassociated distractor is more likely on high-value trials than on low-value trials, because the threshold that the associated saccadic activity has to exceed is reduced relative to low-value trials. On this account, the pairing of a particular stimulus feature with reward does not increase the likelihood for that feature to capture attention and eye movements in the future, per se. Rather, the presence of a reward-associated stimulus lowers the threshold for a saccade to be made to any salient stimulus, regardless of whether it possesses the reward associated feature. Notably, this hypothesis is consistent with findings from primate research, which demonstrate that the expectation of reward disinhibits the activity of neurons in the superior colliculus via feedback connections from rewardprocessing structures such as the basal ganglia (Ikeda \& 
Hikosaka, 2003). In principle, the increased rate of baseline activity resulting from this disinhibition should make it easier for stimulus-evoked activity from other bottom-up inputs to elicit saccades (Vokoun, Mahamed, \& Basso, 2011), effectively lowering the saccade threshold.

A recent study by Failing, Nissens, Pearson, Le Pelley, and Theeuwes (2015) goes some way towards empirically assessing this latter account of VMOC. In this study, participants completed the gaze-contingent visual search task used by Le Pelley et al. (2015), but all stimuli in the search display were rendered in different colours, so that the reward-predicting distractors (red and blue circles) were no longer colour singletons and hence not physically salient. Nevertheless, a VMOC effect occurred; participants were more likely to look at (and hence trigger omission trials for) the high-value distractor than the low-value distractor. Furthermore, the VMOC effect was found to be largest at short saccade latencies - a pattern that is very similar to what has previously been observed in oculomotor capture by physically salient stimuli (e.g., Donk \& van Zoest, 2008). This is consistent with the idea that reward exerts an influence on low-level oculomotor competition in the saccade map. However, because all of the stimuli in the display were nonsalient, we would not expect the reward-associated distractors to generate any more bottom-up saccadic activity than any other stimulus in the display. Thus, while Failing et al.'s findings demonstrate that reward exerts its influence at an earlier stage of processing than goal-directed target selection does, it remains unclear whether reward value and physical salience engage in direct competition on the saccade map such that reward influences the very fastest saccades. That is, rapidly initiated saccades may be driven purely by the physical salience of stimuli in the visual field, whereas slow saccades are influenced by top-down target selection processes, and reward exerts its influence on saccades with an intermediate latency.

The current experiments aimed to establish more clearly whether reward influences early competition on the saccade map, using a variant of Le Pelley et al.'s (2015) VMOC procedure with task-irrelevant distractors. On the majority of trials, the reward-predicting distractors were salient colour singletons, as in our previous work. However, on a subset of trials, participants were presented with both reward-predicting distractors (i.e., the high-value and low-value distractors) in the same search display. Hence, on these "both-distractor" trials, the display contained two task-irrelevant, coloured items of equivalent physical salience. If reward value influences oculomotor capture by reducing the threshold for a bottom-up saccade to a physically salient stimulus, we would expect oculomotor capture to be equally distributed between the two equally salient distractors. If, however, reward value influences competition on the saccade map, gaze should be preferentially captured by the high-value distractor rather than the low-value distractor, on bothdistractor trials. In particular, if this pattern of greater oculomotor capture by the high-value distractor were apparent for the most rapidly initiated saccades, then this would suggest that early competition on the saccade map is modulated by reward prediction and is not merely a function of physical salience.

\section{Experiment 1}

\section{Method}

Participants Previous studies (Failing et al., 2015; Le Pelley et al., 2015; Pearson et al., 2015) have found medium to very large effect sizes for the single-distractor VMOC effect (Cohen's $d \approx 0.54-2.2$ ). Thus, we ran the experiment for as many days as required to test 40 participants, which would give us power of $\sim 0.87$ to detect an effect size of $d=0.5$. In total, 42 UNSW Australia students (mean age $=21.4$, SEM $=$ $0.72,10$ females) participated for course credit. They also received a monetary bonus dependent upon their performance $(M=13.16$ AUD, $S E M=0.43$ AUD). All research reported in this article was approved by the Human Research Ethics Advisory Panel (Psychology) of UNSW Australia.

Apparatus Participants were tested individually using a Tobii TX300 eye tracker, with $300 \mathrm{~Hz}$ temporal and $0.15^{\circ}$ spatial resolution, mounted on a 23 -inch monitor $(1920 \times 1280$ resolution, $60 \mathrm{~Hz}$ refresh rate). Participants' heads were positioned in a chin rest $60 \mathrm{~cm}$ from the screen. For gaze-contingent calculations, the experiment script sampled from the eye tracker every $10 \mathrm{~ms}$, with current gaze location defined as the average gaze location during the preceding $10 \mathrm{~ms}$ sample. The eye tracker was calibrated prior to the practice phase, prior to the main experiment, and twice more during the experiment (after seven blocks, and after 14 blocks). Stimulus presentation was controlled by MATLAB using Psychophysics Toolbox extensions (Brainard, 1997; Kleiner et al., 2007; Pelli, 1997).

Stimuli Each trial consisted of a fixation display, a search display, and a feedback display (see Fig. 1). All stimuli were presented on a black background. The fixation display consisted of a white cross ( 0.5 degrees of visual angle; dva) presented in the centre of the screen, inside a white circle $(3.0 \mathrm{dva})$. The search display consisted of the fixation cross surrounded by six filled shapes $(2.3 \times 2.3 \mathrm{dva})$ equally distributed on an imaginary ring with diameter $10.1 \mathrm{dva}$. The first stimulus was positioned directly above the fixation cross. Five of the shapes were circles (nontargets), and one was a diamond (target). The diamond and three of the circles were always rendered in grey. The remaining two circles were rendered in either red, green, or the same shade of grey as the other shapes (CIE $x, y$, chromaticity coordinates of $.602 / .371$ for red, $.260 / .588$ for green, and $.326 / .388$ for grey) depending on the trial type (see Design section). The values of red and green had similar luminance $\left(\sim 0 \mathrm{~cd} / \mathrm{m}^{2}\right)$, which was higher than that of grey $\left(14.2 \mathrm{~cd} / \mathrm{m}^{2}\right)$. The feedback screen 


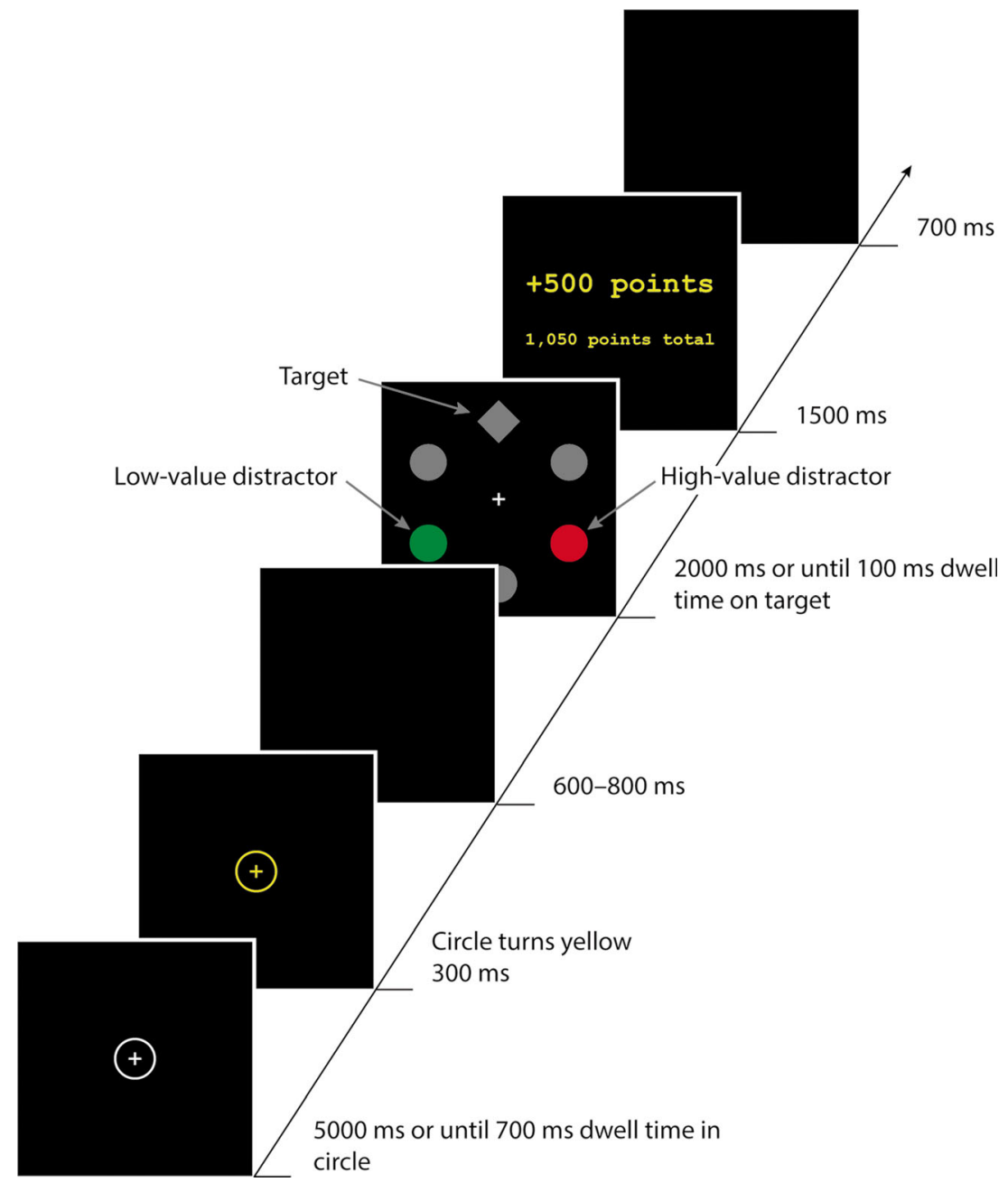

Fig. 1 Sequence of trial events in Experiment 1. Participants responded by moving their eyes to the diamond-shaped target. One of the nontarget circles that was either one or two positions away from the target was designated as the distractor. On both-distractor trials, another nontarget circle that was equidistant from the target was designated as a second distractor. These distractor circles could be rendered in either red or green, depending on the trial type. Fast responses were followed by a reward of points that were converted into money at the end of the experiment. When there was a single distractor rendered in the highvalue colour (high-single distractor trials), large reward was

displayed the points earned on the previous trial as well as the total points accumulated in the experiment. If response time (RT) was greater than the soft-timeout threshold (see below), the message "Too slow" appeared below the feedback that the reward was 0 points for the trial.

Design For half of the participants, red was the high-value colour and green was the low-value colour; these colour-reward relationships were reversed for the other half of participants. There were four different types of trial: (1) trials in which one of the nontarget circles was rendered in the highvalue colour (henceforth referred to as high-single distractor trials), (2) trials in which one of the nontarget circles was available for a fast eye movement to the target. When there was a single distractor rendered in the low-value colour (low-single distractor trials), small reward was available. When one distractor was rendered in the high-value colour and another distractor was rendered in the low-value colour (both-distractor trials), or when no distractor was present in the display (distractor-absent trials), large and small reward were equally likely. If gaze was recorded within a region of interest surrounding the distractor(s) prior to being registered on the target, an omission was triggered and no reward was delivered. (Colour figure online.)

rendered in the low-value colour (low-single distractor trials), (3) trials in which all nontarget circles were rendered in grey (distractor-absent trials), and (4) trials in which one of the nontarget circles (the high-value distractor) was rendered in the high-value colour and another non-target circle (the lowvalue distractor) was rendered in the low-value colour (bothdistractor trials). The experiment comprised 21 blocks of 34 trials each, for a total of 714 experimental trials. Each block consisted of 12 high-single distractor trials, 12 low-single distractor trials, six both-distractor trials, and four distractorabsent trials, in random order.

On each trial, the location of the target was randomly determined. On high-single and low-single distractor trials, the 
location of the distractor was random with the constraint that it was never positioned directly opposite the target but was either one or two positions away (i.e., the polar angle between the target and the distractor was either $60^{\circ}$ or $120^{\circ}$ ). On bothdistractor trials, the high-value distractor was positioned with the same constraints as above, and the low-value distractor was positioned so as to be the same distance from the target as the high-value distractor (i.e., if the high-value distractor was $60^{\circ}$ from the target in the clockwise direction, the lowvalue distractor would be $60^{\circ}$ from the target in the anticlockwise direction).

A small circular region of interest (ROI) with diameter 3.5 dva was defined around the centre of the diamond target; a larger ROI (diameter $5.1 \mathrm{dva}$ ) was defined around each of the distractors. A response was registered after the participant accumulated $100 \mathrm{~ms}$ of gaze dwell time within the target ROI. Responses with RTs that were slower than the softtimeout threshold were not rewarded; this threshold was $800 \mathrm{~ms}$ for the first training block and $600 \mathrm{~ms}$ for the subsequent blocks. If ever any gaze was detected within one of the distractor ROIs, the trial was recorded as an omission trial and the reward was not delivered. On distractor-absent trials, one of the nontarget circles (that was either one or two positions away from the target) was selected at random to act as the omission-triggering location; gaze falling within an ROI surrounding the selected grey circle triggered an omission in exactly the same way as if it were a distractor.

On each trial, reward was delivered if RT was faster than the soft-timeout threshold and an omission trial had not been triggered: 500 points on high-single distractor trials, 10 points on low-single distractor trials, and an equal likelihood of 500 points or 10 points on both-distractor trials ${ }^{1}$ and distractor-absent trials.

Procedure Participants were told that their task was to move their eyes to the diamond shape on each trial and that they could earn either 0 points, 10 points, or 500 points "depending on how fast and accurate" their response was. They were

\footnotetext{
${ }^{1}$ The reward outcome on both-distractor trials was randomly set to either the high-value reward or the low-value reward rather than the sum of the high- and low-value rewards. If participants were to consistently receive the sum of both reward values on these trials, any pattern of oculomotor capture observed may be the consequence of this new learned relationship (i.e., two distractors $=$ very large reward) rather than the participant's prior experience with the stimuli. By randomly giving either the high- or lowvalue reward, the presentation of two distractor stimuli in the same display is nonpredictive of reward magnitude, and any oculomotor bias must be governed by the participant's prior learning. This is related to the reason why there were relatively few both-distractor trials in each block (only 6, as compared to 12 single-distractor trials). On both-distractor trials, participants could receive a high-value reward even though a low-value distractor was present in the display, and vice versa. By having relatively few both-distractor trials, our aim was to minimise the extent to which such experiences would "water down" the prevailing relationship between each distractor colour and its associated reward established on single-distractor trials.
}

informed that the points earned during the task would determine the monetary reward they received at the end of the experiment and that most participants could earn between 7 and 15 AUD for good performance (no specific information was given about the conversion rate from points to AUD). The session began with eight unrewarded practice trials that contained a yellow distractor, followed by the experimental trials. Participants took a short rest break every 68 trials.

Each trial began with the presentation of the fixation display. Participants' gaze location was superimposed on the display as a small yellow dot. Once $700 \mathrm{~ms}$ of gaze dwell time had been recorded within the circle surrounding the fixation cross, or after $5 \mathrm{~s}$, the cross and the circle turned yellow, and the dot marking the participant's gaze location disappeared. After $300 \mathrm{~ms}$ the screen went blank, and after a random period of 600,700 , or $800 \mathrm{~ms}$, the search display appeared. The trial terminated after a response was recorded (see Design), or after 2 s (hard timeout) had passed. The feedback display then appeared and remained onscreen for 2,500 $\mathrm{ms}$ in the first experimental block, and 1,500 $\mathrm{ms}$ in all subsequent blocks. The intertrial interval was $700 \mathrm{~ms}$.

Data analysis Following previous protocols (Le Pelley et al., 2015; Pearson et al., 2015), data from the first two experimental trials and the first two trials after each break were discarded. Hard timeouts (1.8\% of all trials) were also discarded. For the remaining trials, averaging across all participants, valid gaze location data were registered in $97.0 \%$ (SEM $0.6 \%$ ) of all samples. This suggests very high fidelity of gaze data on these trials.

For the analysis of saccade latencies (i.e., the time between the presentation of the search display and the initiation of the first saccade), a velocity-threshold identification (I-VT) algorithm (Salvucci \& Goldberg, 2000) with a velocity criterion of $30 \mathrm{dva} / \mathrm{s}$ was used to detect saccades in the raw data from the eye tracker (sampled at $300 \mathrm{~Hz}$ rather than $100 \mathrm{~Hz}$ used for the gaze-contingent calculations). For these analyses (and again following Le Pelley et al., 2015), in addition to the exclusions described previously, we further excluded all trials in which the latency of the first saccade after the presentation of the search display was less than $80 \mathrm{~ms}$ (anticipatory saccades; $12.3 \%$ of all trials) or no gaze was recorded within 5.1 dva (100 pixels) of the fixation point within the first $80 \mathrm{~ms}$ (7.5\% of all trials).

\section{Results}

Omission trials Figure 2A shows the proportion of omission trials averaged across all blocks. These data were analysed using a one-way (trial type: high-single, lowsingle, both, absent) analysis of variance (ANOVA), which found a significant main effect of trial type, $F(3$, 
Fig. 2 Eye gaze and saccade latency data from Experiment 1. (A) Mean proportion of omission trials, averaged across blocks, for high-single, low-single, bothdistractor, and distractor-absent trials. (B) Mean proportion of both-distractor trials with gaze on the high-value distractor and lowvalue distractor. (C) Mean saccade latencies for saccades toward the target and distractor, averaged across blocks. (D) Mean saccade latencies for saccades made toward the target, the highvalue and low-value distractor on both-distractor trials. All error bars show within-subjects SEM (Cousineau, 2005). (Colour figure online.)

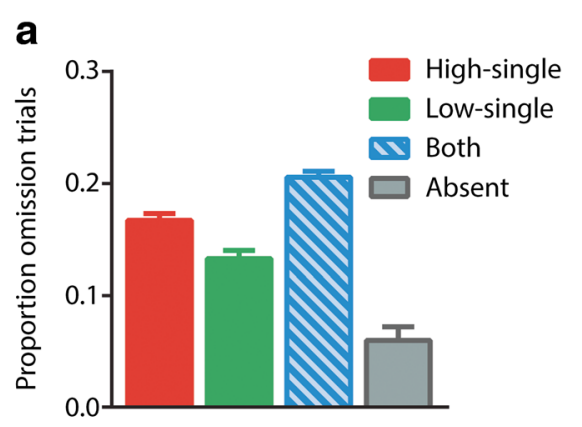

C

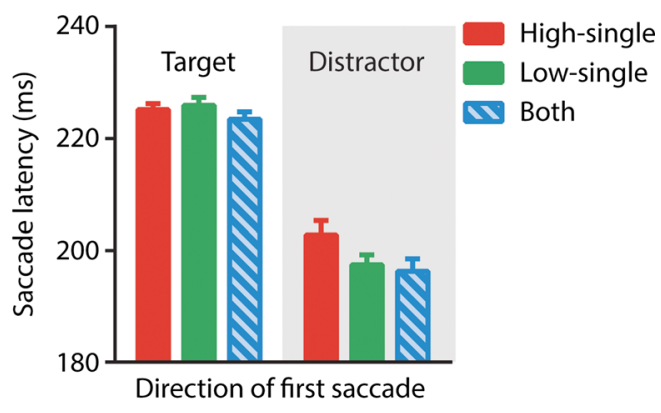

d

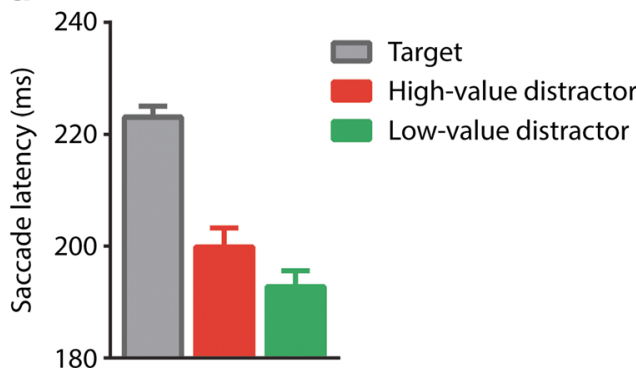

123) $=50.4, p<.001, \eta_{\mathrm{p}}^{2}=.55$. Planned pairwise $t$ tests were used to further explore this effect. More omissions were triggered on each of the trial types that contained a salient distractor than on distractor-absent trials-highsingle versus absent: $t(41)=7.39, p<.001, d=1.14$; low-single versus absent: $t(41)=6.53, p<.001, d=$ 1.01 ; both versus absent: $t(41)=9.74, p<.001, d=$ 1.50. Furthermore, more omissions were triggered on both-distractor trials (where gaze on either distractor could trigger an omission) than on trials that contained a single distractor-both versus high-single: $t(41)=4.42, p$ $<.001, d=.68$; both versus low-single: $t(41)=6.40, p<$ $.001, d=.99$. Finally, and most importantly, omissions were more likely on trials that contained the high-value distractor alone than trials that contained the low-value distractor alone, $t(41)=2.82, p=.007, d=.44$, demonstrating a significant VMOC effect.

Distribution of gaze on both distractor trials The difference in proportion of omissions on high-single distractor versus low-single distractor trials replicates the findings of Le Pelley et al. (2015). Having verified that this procedure produced a VMOC effect, we turned to the analysis of the bothdistractor trials in order to determine whether reward-value information biases competition on the saccade map. Crucially, Fig. 2B shows that participants looked at the highvalue distractor more often than the low-value distractor on both-distractor trials, and a paired $t$ test revealed that this difference was significant, $t(41)=2.22, p=.032, d=.34$.
Correlation of single-distractor VMOC effect with bothdistractor VMOC effect The difference in proportion on omission trials on high-single versus low-single distractor trials provides one measure of the influence of value on oculomotor capture (we term this the single-distractor VMOC

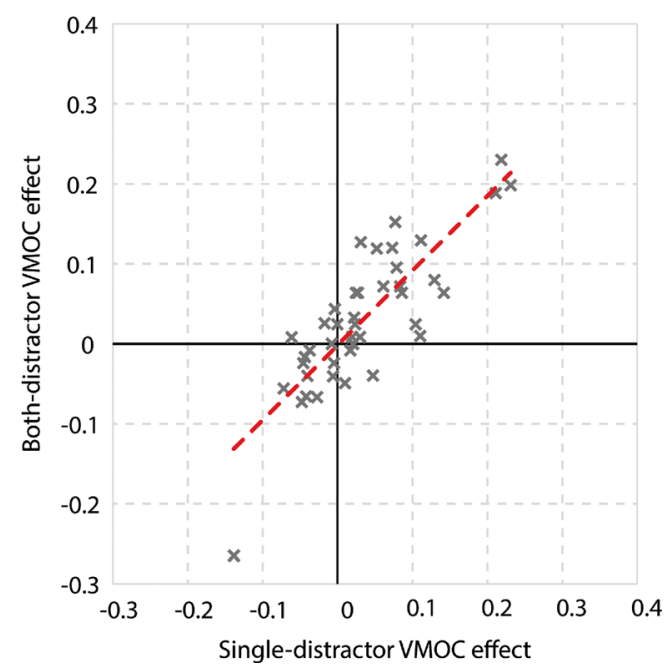

Fig. 3 Scatterplot of single-distractor VMOC effect (horizontal axis; calculated as proportion of omissions on high-single distractor trials minus proportion of omissions on low-single distractor trials) against both-distractor VMOC effect (vertical axis; calculated as proportion of both-distractor trials with gaze on the high-value distractor minus the proportion of both-distractor trials with gaze on the low-value) distractor in Experiment 1. Dotted line shows line of best linear fit. (Colour figure online.) 
effect). The difference in proportion of both-distractor trials with gaze on the high-value distractor versus the low-value distractor provides a second, independent measure (bothdistractor VMOC effect). The scatterplot in Fig. 3 shows a strong positive correlation between these two measures, Pearson's $r(40)=.836, p<.001$. That is, participants who showed a larger difference between proportion of omissions on high-single and low-single distractor trials (i.e., those who displayed a larger single-distractor VMOC effect) tended to show more oculomotor capture by the high-value distractor than the low-value distractor when both stimuli were presented simultaneously.

Latency of first saccades Preliminary analysis showed that latency of first saccades did not change significantly over the course of the experiment, with no evidence of a linear trend across blocks, $F(1,39)<1$. Subsequent analyses therefore collapsed across blocks. Figure 2C shows mean latency of the first saccade for each trial type as a function of saccade direction. Saccades were defined as going in the direction of the target or the distractor if the endpoint of the saccade had an angular deviation of less than $30^{\circ}$ to the left or right (i.e., half the distance between the stimuli in the display) of the critical stimulus. The data were analysed using a 3 (trial type: highsingle, low-single, both) $\times 2$ (saccade direction: target, distractor) ANOVA. This showed a significant main effect of direction, $F(1,41)=194.3, p<.001, \eta_{\mathrm{p}}^{2}=.83$, with shorter latency for saccades going to the distractor than those going to the target. The main effect of trial type, $F(2,82)=2.22, p=$ $.115, \eta_{\mathrm{p}}^{2}=.05$, and trial type $\times$ direction interaction, $F(2,82)$ $=.82, p=.444, \eta_{\mathrm{p}}^{2}=.02$, were both nonsignificant. Paired $t$ tests found no evidence that the presence of at least one physically salient distractor in the display had an effect on the latency of saccades to the target - absent versus high-single, $t(41)=.577, p=.567, d=.11$; absent versus low-single, $t(41)$ $=.876, p=.386, d=.14$; absent versus both, $t(41)=.718, p=$ $.477, d=.11$. Figure 2D shows a breakdown of first saccade latencies on both-distractor trials according to whether they were towards the target, the high-value distractor, or the low-value distractor. Two participants did not make any initial saccades towards one of the distractors and so were removed from subsequent analyses (remaining $n=40$ ). As above, latencies of saccades going towards the target were significantly longer than those towards either of the distractors on these trials - target versus high-value distractor, $t(39)=5.11, p<$ $.001, d=0.81$; target versus low-value distractor, $t(39)=$ $8.18, p<.001, d=1.29$. There was no significant difference in the latency of saccades going to the high-value and lowvalue distractors, $t(39)=.49, p=.63, d=.08$.

Time course of the both-distractor VMOC effect In order to investigate the time course of the VMOC effect on both- distractor trials, we analysed the proportion of first saccades going towards the high-value and low-value distractors on both-distractor trials as a function of saccade latency using the Vincentizing procedure (Ratcliff, 1979). We calculated mean first saccade latencies and the proportion of first saccades going towards each distractor separately for each decile of the individual saccade latency distributions (see Fig. 4). The data were initially analysed using a 2 (distractor: highvalue, low-value $) \times 10$ (decile) ANOVA. This found a significant main effect of distractor, $F(1,41)=4.54, p=.039, \eta_{\mathrm{p}}^{2}=$ .10 , such that more first saccades went to the high-value distractor than the low-value distractor, averaged across saccade latency. There was also a significant main effect of decile, $F(9,369)=19.83, p<.001, \eta_{\mathrm{p}}^{2}=.33$, indicating that the proportion of saccades going towards either distractor decreased as a function of saccade latency. The distractor $x$ decile interaction was nonsignificant, $F(9,369)=.66, p=$ $.748, \eta_{\mathrm{p}}^{2}=.02$. To determine whether the both-distractor VMOC effect was evident for particularly rapid saccades, a planned, paired samples $t$ test was used to compare the proportion of first saccades going towards the high-value distractor and low-value distractor for the fastest decile of saccades. This revealed a marginally significant difference, $t(41)=1.72, p=.093, d=.27$.

\section{Discussion}

In Experiment 1, participants were more likely to have their gaze captured by the high-value distractor than the low-value distractor, even though looking at the distractor stimuli was directly counterproductive to the participant's goals, because it resulted in the omission of the reward that would otherwise have been delivered on that trial. This is a replication of the

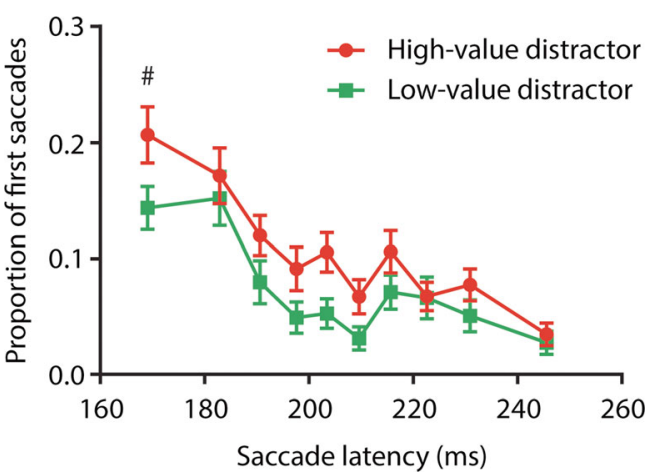

Fig. 4 The proportion of first saccades towards the high-value and lowvalue distractor on both-distractor trials as a function of first saccade latency decile in Experiment 1. Mean first saccade latencies and the proportion of first saccades directed toward each distractor were calculated separately for each decile of individual participant saccade latency distributions using the Vincentizing procedure (Ratcliff, 1979). $\# p<.10$. Error bars show within-subjects SEM. (Colour figure online.) 
VMOC effect that we have previously reported (Failing et al., 2015; Le Pelley et al., 2015; Pearson et al., 2015). The important novel finding of this experiment is that the VMOC effect was also evident when both the high- and low-value distractors were present in the same search display. We take this as evidence that reward value engages in competition with stimulus-driven and goal-directed inputs to the oculomotor system on a common saccadic priority map. In a time-course analysis, there was a trend towards this both-distractor VMOC effect being present in the shortest decile of saccade latencies, which suggests that reward information may influence competition on the saccade map even at particularly early stages of processing. Furthermore, the both-distractor VMOC effect was highly correlated with the VMOC effect evident on single-distractor trials, which suggests that the same processes are likely to be responsible for both effects.

However, some aspects of the data from Experiment 1 bear further consideration. First, although the mean latency of saccades directed towards either of the distractors was shorter than that of saccades directed towards the target (suggesting that the stimulus-driven activity associated with the physically salient distractor reached the saccade threshold faster than the goal-directed activity associated with selection of the less salient target; Godijn \& Theeuwes, 2002; cf. Walker, Walker, Husain, \& Kennard, 2000), there was no effect of reward value on saccade latencies to either the target or to the distractor (see Fig. 2). We return to this null finding in the General Discussion.

Second, the results of the time-course analysis of the VMOC effect on both-distractor trials were not clear-cut. Averaging across all deciles, significantly more first saccades was directed towards the high-value distractor than the low-value distractor. However, when considering just the shortest decile of saccade latencies, this trend reached only marginal significance. Furthermore, there was no significant interaction between the effect of distractor type and decile (i.e., the numerical trend suggesting a reduction in the influence of reward as decile increased did not reach significance, see Fig. 4). It is possible that the somewhat equivocal findings relating to time course reflect a lack of power in Experiment 1; participants experienced 126 both-distractor trials over the course of the whole experiment, meaning that the data for each decile of the saccade latency distribution correspond to only around 10-11 trials for each participant (given the data exclusions detailed above). Moreover, many of these trials would have occurred early in the experiment, before participants had much experience of the colour-reward relationships that drive the VMOC effect, thus reducing the power of the time-course analysis of this effect still further. We therefore ran a second experiment in which we increased the number of both-distractor trials, in order to enhance the sensitivity of the time-course analysis.

\section{Experiment 2}

Experiment 2 was effectively an extended version of Experiment 1, in which participants completed 1,260 trials - including 294 both-distractor trials - over the course of two sessions run on consecutive days (as compared to 714 trials - with 126 both-distractor trials - in a single session in Experiment 1). As the primary aim of Experiment 2 was to collect more data relating to saccades towards reward-related distractors, distractor-absent trials were removed. In an attempt to reduce the number of trials in which an anticipatory saccade was made, or in which no valid gaze samples were recorded in the centre of the screen at the start of the trial, the time between the offset of the fixation cross and the presentation of the search display was reduced to $150 \mathrm{~ms}$, in keeping with previous studies that have used similar saccade latency analyses (e.g., Failing et al., 2015).

\section{Method}

Participants Thirty-eight participants (mean age $=21.7$, SEM $=0.75,24$ females) took part in Experiment 2, either for course credit $(n=15)$ or for payment of 30 AUD $(n=23)$. All participants received an additional monetary bonus that was dependent upon their performance ( $M=15.05$ AUD, $S E M=0.36$ AUD).

Apparatus and stimuli The apparatus and stimuli were identical to those of Experiment 1, with one exception. Instead of the reward-associated distractor circles being rendered in either red or green, in Experiment 2 they were rendered in either red or blue (CIE, $x, y$, chromaticity coordinates of $.595 / .360$ for red, .160/.116 for blue). The luminance of the red and blue stimuli was similar $\left(\sim 42.5 \mathrm{~cd} / \mathrm{m}^{2}\right)$, which was higher than that of the grey used for all other shapes $\left(14.2 \mathrm{~cd} / \mathrm{m}^{2}\right)$.

Design The experiment comprised 42 blocks of 30 trials each for a total of 1,260 experimental trials, which were completed over the course of two 1-hr sessions run on consecutive days. In the first session, each block consisted of 12 high-single distractor trials, 12 low-single distractor trials, and six bothdistractor trials. For the second session, each block consisted of 11 high-single distractor trials, 11 low-single distractor trials, and eight both-distractor trials. The proportion of bothdistractor trials was thus increased slightly in the second session, providing more trials from a period in which participants had had extensive experience of the colour-reward relationships, and so increasing the sensitivity of the time-course analysis of the both-distractor VMOC effect. There were no distractor-absent trials. All other aspects of the design were as for Experiment 1. 
Procedure The general procedure for each session was the same as in Experiment 1. The only differences were that, in Experiment 2, (1) the fixation cross was not presented as part of the search display, and (2) the pause between the offset of the fixation display and the presentation of the search display was reduced to $150 \mathrm{~ms}$.

Data analysis The same data analysis protocol was used as in Experiment 1 . Hard timeouts ( $0.9 \%$ of trials) were discarded. Across the remaining trials, valid gaze location data were registered in $96.6 \%(S E M=1.3 \%)$ of samples. For the analysis of saccade latency and the time course of oculomotor capture, $12.1 \%$ of trials were discarded due to the presence of anticipatory saccades, and $6.5 \%$ of trials were discarded due to no valid gaze samples being recorded within $5.1 \mathrm{dva}$ (100 pixels) of the fixation point within the first $80 \mathrm{~ms}$.

\section{Results}

Omission trials Figure 5A shows the proportion of omission trials for each trial type averaged across all blocks in the experiment. These data were analysed using a one-way (trial type: high-single, low-single, both) ANOVA, which found a significant main effect of trial type, $F(2,74)=30.6, p<.001$, $\eta_{\mathrm{p}}^{2}=.45$. In order to further explore this effect, planned pairwise $t$ tests were conducted. As was the case in Experiment 1, more omissions were triggered on bothdistractor trials than trials that contained a single salient distractor-both versus high-single: $t(37)=7.22, p<.001, d$ $=1.17$; both versus low-single: $t(37)=6.95, p<.001, d=$ 1.13. More importantly, there was also a significant singledistractor VMOC effect, with participants making more omissions on high-single trials than low-single trials, $t(37)=2.51$, $p=.017, d=.41$.

Distribution of gaze on both-distractor trials Having confirmed the presence of a single-distractor VMOC effect, we turned to the analysis of both-distractor trials. As can be seen in Fig. 5B, a both-distractor VMOC effect was once again evident, with participants looking at the high-value distractor more often than the low-value distractor on both-distractor trials, $t(37)=2.76, p=.009, d=.45$.

Correlation of single-distractor VMOC effect with bothdistractor VMOC effect The single- and both-distractor VMOC effects were quantified as for Experiment 1. Figure 6 shows a strong positive correlation between these measures, $r(36)=.923, p<.001$.

Latency of first saccades Figure 5C shows the average latency of the first saccade for each trial type when it was directed towards the target and when it was directed towards the distractor. A 3 (trial type: high-single, low-single, both) $\times 2$ (direction: target, distractor) ANOVA found a significant main effect of direction, $F(1,37)=276.6, p<.001$, $\eta_{\mathrm{p}}^{2}=.88$, such that first saccades made towards distractors had a shorter
Fig. 5 Eye gaze and saccade latency data from Experiment 2. (A) Mean proportion of omission trials, averaged across all blocks, for high-single, low-single, and both-distractor trials. (B) Mean proportion of both-distractor trials with gaze on the high-value distractor and low-value distractor. (C) Mean saccade latencies for saccades towards the target and distractor. (D) Mean saccade latencies for saccades made to the target, the high-value and low-value distractor on bothdistractor trials. Error bars show within-subjects SEM. (Colour figure online.)
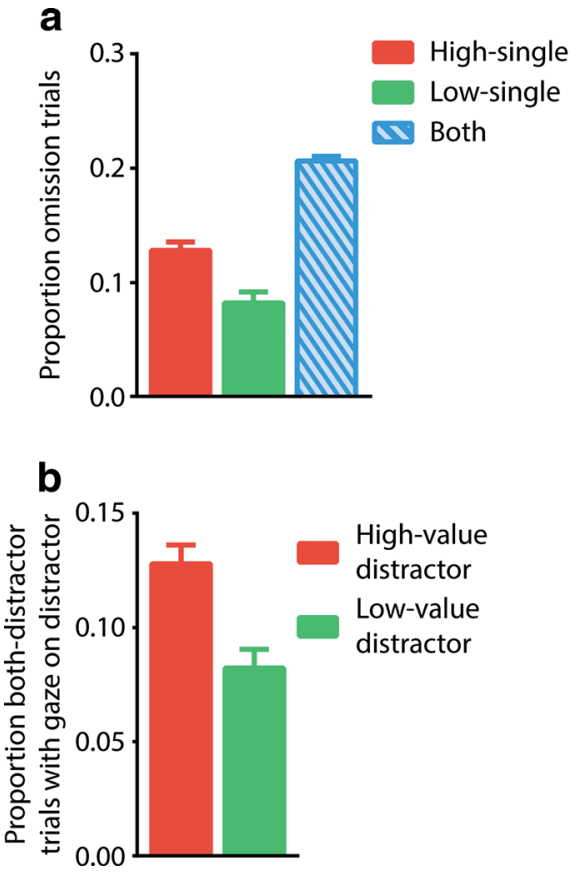

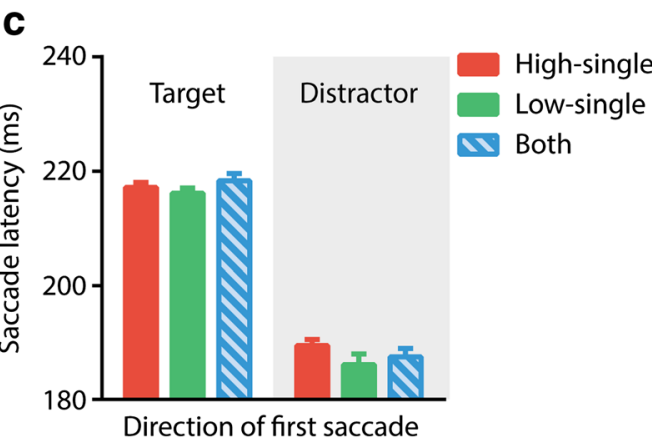

d

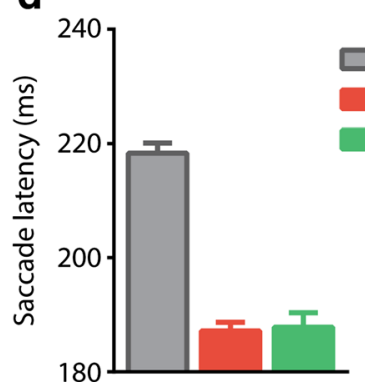


latency than those towards the target. The main effect of trial type, $F(2,74)=1.99, p=.143, \eta_{\mathrm{p}}^{2}=.05$, and the trial type $\times$ direction interaction, $F(2,74)=.98, p=.379, \eta_{\mathrm{p}}^{2}=.03$, were both nonsignificant. Subsequent paired $t$ tests revealed no significant difference between high-single and low-single trials in terms of the latency of first saccades directed towards the target, $t(37)=1.30, p=.201, d=.21$, or towards the distractor, $t(37)=1.48, p=.147, d=.24$.

Figure 5D shows first saccade latencies on both-distractor trials according to whether their endpoint was in the direction of the target, the high-value distractor, or the low-value distractor. In line with the previous analyses, saccades were slower when they were directed towards the target than when directed towards either of the distractors - target versus highvalue distractor, $t(37)=13.0, p<.001, d=2.11$, target versus low-value distractor, $t(37)=7.01, p<.001, d=1.14$. There was no difference in the latency of first saccades that were directed to the high-value distractor versus those directed to the low-value distractor, $t(37)=0.17, p=.863, d=.02$.

Time course of both-distractor VMOC effect To assess whether reward value asserts its influence on oculomotor selection at an early stage of processing, we analysed the proportion of first saccades going towards the high-value and low-value distractor on both-distractor trials as a function of their saccade latency using the Vincentizing procedure (see Fig. 7). The data were analysed using a 2 (distractor: highvalue, low-value $) \times 10$ (decile) ANOVA. $^{2}$ This revealed a main effect of distractor, $F(1,37)=10.41, p=.003, \eta_{\mathrm{p}}^{2}=$ .22 , with more first saccades directed towards the high-value distractor than the low-value distractor on average. The main

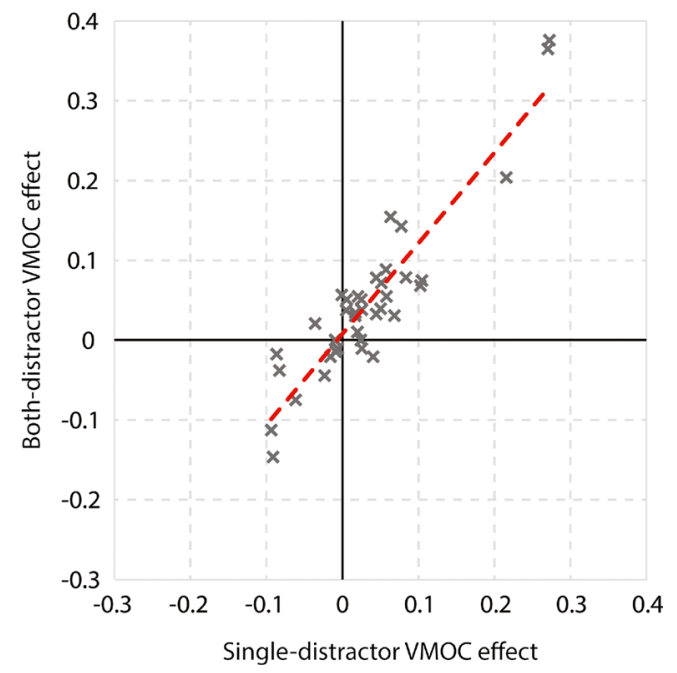

Fig. 6 Scatterplot of single-distractor VMOC effect (horizontal axis) against both-distractor VMOC effect (vertical axis) distractor in Experiment 2. Dotted line shows line of best linear fit. (Colour figure online.) effect of decile was also significant, $F(9,333)=45.66, p<$ $.001, \eta_{\mathrm{p}}^{2}=.55$, with the proportion of first saccades directed towards either distractor decreasing as saccade latency increased. Crucially, the distractor $\times$ decile interaction was significant, $F(9,333)=3.07, p=.001, \eta_{\mathrm{p}}^{2}=.08$, and demonstrated a significant linear trend, $F(1,37)=11.05, p=.002, \eta_{\mathrm{p}}^{2}=$ .23 , indicating that the effect of reward on saccade direction was greatest for the fastest saccades, and decreased proportionately as saccade latency increased. Notably, the pattern of more eye-movements towards the high-value distractor than the low-value distractor was significant for the shortest latency decile, $t(37)=2.61, p=.013, d=.42$.

\section{Discussion}

Experiment 2 replicated the key findings of Experiment 1. Once again, task-irrelevant stimuli associated with highvalue rewards were more likely to capture attention than stimuli associated with low-value rewards, even when both stimuli were presented in the same search display. Importantly, the time-course analysis demonstrated that the both-distractor VMOC effect was strongest for the most rapidly initiated saccades and decreased linearly as saccade latency became longer. This suggests that reward value influences competition on the saccade map at a particularly early stage of processing. This pattern was similar to that observed in Experiment 1, though both of the important effects (the distractor $\times$ decile interaction, and the effect of distractor at the shortest decile) reached full significance in Experiment 2. This is most likely a consequence of greater power in Experiment 2, for which the time-course analysis included over twice as many bothdistractor trials as in Experiment 1 (294 vs. 126). However, despite the greater power of Experiment 2, we still failed to observe an effect of reward on the latency of saccades directed towards either the target or the distractor (see Fig. 5). We take up this issue again in the General Discussion.

\section{General discussion}

In two experiments, participants were trained that the presence of a distractor stimulus with a particular colour in a visual search display signalled the magnitude of reward that was available for a rapid saccade towards a shape singleton target. The reward-signalling distractor was task-irrelevant throughout each experiment, given that the participant's goal was to

\footnotetext{
${ }^{2}$ The same pattern of significant results was observed when session was included as a factor in the ANOVA (recall that Experiment 2 involved two sessions, conducted on successive days). Furthermore, we did not find any evidence of a three-way Session $\times$ Distractor $\times$ Decile interaction $(F<$ $1)$, suggesting that there was no difference in the time-course of the bothdistractor VMOC effect between sessions.
} 


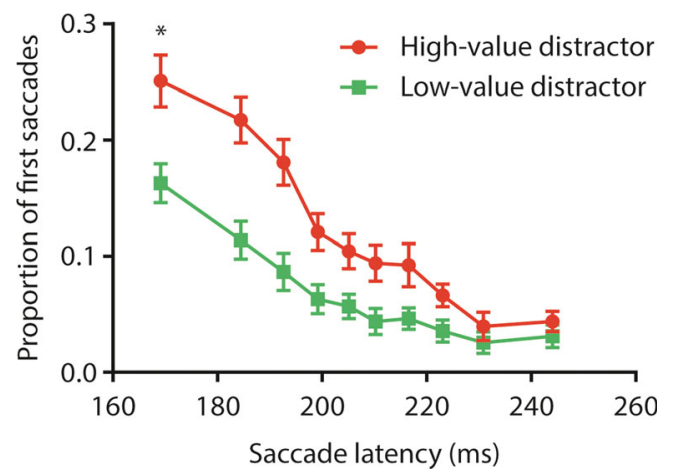

Fig. 7 The proportion of first saccades toward the high-value and lowvalue distractor on both-distractor trials as a function of first saccade latency decile in Experiment 2. ${ }^{*} p<.05$. Error bars show withinsubjects SEM. (Colour figure online.)

look at the target. Indeed, looking at the distractor was directly counterproductive, as the reward for the trial was omitted if gaze was detected in its vicinity. Nevertheless, participants made more eye movements to a distractor that signalled high reward than a distractor that signalled low reward. This is a replication of the value-modulated oculomotor capture (VMOC) effect that we have observed previously (Failing et al., 2015; Le Pelley et al., 2015; Pearson et al., 2015). The important novel finding of the present studies is that the VMOC effect was also evident when both the high- and low-value distractors were presented in the same search display. On these both-distractor trials, participants again made more eye movements to the high-value distractor than to the low-value distractor. We take this as evidence that effects of reward are integrated (and compete) with stimulus-driven and goal-directed inputs to the oculomotor system on a common saccadic priority map. Notably, this both-distractor VMOC effect was evident at the shortest saccade latencies, suggesting that reward information is incorporated on the saccade map at a particularly early stage of processing. Furthermore, the both-distractor VMOC effect was highly correlated with the VMOC effect evident on single-distractor trials, which suggests that the same processes are likely to be responsible for both effects.

These findings have interesting implications for our understanding of the mechanisms underlying VMOC. A number of previous studies have argued that reward value exerts its influence on visual selection at the early stages of processing (e.g., Anderson et al., 2011a, 2011b; Anderson \& Yantis, 2012; Awh et al., 2012; Belopolsky, 2015; Theeuwes \& Belopolsky, 2012; Hickey et al., 2010, 2011). For example, Belopolsky (2015) recently suggested that a number of automatic biases that develop based on prior experience with a stimulus (e.g., reward history, selection history, and emotion) engage in competition with goal-directed and stimulus-driven inputs to the oculomotor system on the saccade map through a process of competitive integration (Godijn \& Theeuwes, 2002). According to this account, activity on the saccade map is enhanced or inhibited according to prior experience with the stimulus features in the display, such that there would be a boost in activity at a location containing a distractor rendered in a colour that has been associated with a relatively high-value reward. Thus, the saccadic activity at this location is more likely to achieve the threshold for a saccade to be initiated relative to an equally salient stimulus that has been paired with low-value reward. As a result, more (erroneous) saccades to the distractor will be made on high-value trials than low-value trials.

However, up to this point, direct evidence that the VMOC effect reflects competition on the saccade map has been lacking. The majority of previous studies have used designs in which the critical stimuli that were shown to involuntarily capture attention and/or eye movements when presented as distractors during a test phase, were task-relevant during an initial pretraining phase (e.g., Anderson et al., 2011a, 2011b; Anderson \& Yantis, 2012; Theeuwes \& Belopolsky, 2012; see also Hickey et al., 2010). That is, participants were required to rapidly orient their attention and/or gaze to the critical stimuli in order to earn the reward that they signalled. Under these conditions, the increased rate of capture by reward-related stimuli may not reflect augmented activity on the saccade map but may instead be a consequence of an overlearned orienting response developed during the pretraining phase (e.g., "saccade to red for large reward") that continues to be habitually enacted during the test phase. Le Pelley et al. (2015; see also Pearson et al., 2015) addressed this potential confound by demonstrating an increased likelihood of oculomotor capture by stimuli that were associated with high-value rewards yet were never task-relevant to participants. However, in this design, the reward-predicting distractors were colour singletons, and so were likely to automatically capture attention and eye movements on the basis of their physical salience alone (Theeuwes et al., 1999; Theeuwes, 1992, 1994). Therefore, it is possible that the VMOC effect observed by Le Pelley et al. was not due to augmentation of the reward-related stimulus feature's activity on the saccade map but rather was a consequence of reward value reducing the global threshold for a saccade to be made to physically salient stimuli. According to this account, when a stimulus feature that has been associated with high-value reward is detected by the visual system, the threshold required for bottom-up saccadic activity to initiate a saccade is lowered. Thus, on high-value trials, the bottom-up activity associated with the colour-singleton distractor is more likely to exceed this reduced threshold, and so more erroneous saccades will be made to the distractor than on low-value trials.

The current study provides a direct test of whether VMOC is driven by reward information biasing competition on the saccade map. As in Le Pelley et al. (2015), participants were trained on a task in which the reward-associated distractor 
stimuli were never task relevant, and so the VMOC effect cannot be a consequence of overtrained oculomotor orienting. Furthermore, on both-distractor trials, two equally salient reward-associated distractors were simultaneously presented in the same search display. Crucially, we would predict a different outcome on these trials, depending on whether reward information is directly integrated onto the saccade map or alternatively lowers the global saccadic threshold. According to the "global threshold" account, on both-distractor trials the presence of a cue that signals high reward should lead to a lowered saccade threshold, but as both of the distractor stimuli are (equally) physically salient, they should be equally likely to capture eye-movements. In contrast, the "competitive integration" account correctly predicts more oculomotor capture by the high-value distractor than the low-value distractor on both-distractor trials because the saccadic activity associated with the high-value distractor location is augmented relative to that of the low-value distractor. Thus, the current study provides direct evidence for reward information biasing competition on the saccade map. This conclusion is further supported by the finding that the both-distractor VMOC effect was most pronounced for rapidly initiated saccades and decreased as a function of first saccade latency. This fits with the idea that reward value exerts its influence early on in the selection process, either by augmenting or engaging in direct competition with the saccadic activity generated by bottom-up salience.

The current findings are consistent with those of a recent study by Failing et al. (2015), which found a VMOC effect using a version of Le Pelley et al.'s (2015) procedure, in which all of the stimuli in the search display were rendered in different colours. Using the Vincentization analysis that was employed in the current study, Failing et al. demonstrated that VMOC by nonsalient stimuli was most prominent for rapid saccades and decreased as a function of saccade latency. Therefore, it was suggested that the VMOC effect was a consequence of reward value being competitively integrated on the saccade map. However, there was a notable procedural difference between the task used by Failing et al. and that reported here (as well as previously: Le Pelley et al., 2015; Pearson et al., 2015). In Failing et al.'s study, participants were explicitly informed prior to the experiment that the search display would always contain either a red or blue nontarget circle, and that the colour of this circle would determine the size of the reward that was available for responding to the target. This raises the possibility that the VMOC effect observed was not a consequence of trial-by-trial reward learning but rather reward modulation of a top-down attentional set for red and blue items. While this explanation is perhaps unlikely given that the VMOC effect was found to be strongest for relatively rapid saccades - whereas top-down influences are typically considered to affect longer-latency saccades (Godijn \& Theeuwes, 2002; Mulckhuyse et al., 2008) - the current study gets around this potential confound by not providing participants with any explicit information about the significance of the reward-predicting distractors in the task. Thus, the current data make clear that experience of the statistical cooccurrence of reward and a stimulus feature is sufficient to bias competition on the saccade map.

More importantly, because the reward-predicting distractors were not distinguished by their physical salience in Failing et al.'s design, it was not possible to determine whether reward information and physical salience engage in direct competition with one another on the saccade map. That is, Failing et al.'s procedure leaves open the possibility that the very fastest saccades are driven purely by physical salience, with reward exerting its effect only for somewhat slower saccades that occur when there is no physically salient stimulus present. Notably, the mean latency of the shortest decile of saccades recorded by Failing et al. ( $\sim 215 \mathrm{~ms})$ would fall at the sixth or seventh decile in the current experiments, in which the distractor is distinguished by its physical salience. However, the current finding of a VMOC effect for the shortest latency saccades to physically salient, reward-related distractors (mean latency $\sim 170 \mathrm{~ms}$ ) rules out this possibility and shows clearly that reward can bias competition at a particularly early stage in processing, by either enhancing or inhibiting the saccadic activity generated by the bottom-up salience of a stimulus.

Although our data suggest that reward value biases activity on the saccade map, there are a number of possible mechanisms by which this may occur. For example, Belopolsky (2015) proposed a nonmodular view of the oculomotor system, in which reward information from various independent inputs (e.g., basal ganglia, frontal eye fields, lateral intraparietal cortex) augments or decreases activity on an integrated attentional network that consists of several priority maps located in different areas of the brain. Alternatively, Hickey et al. $(2010,2011)$ have argued that reward value biases oculomotor competition by enhancing low-level sensory inputs - effectively increasing the perceptual salience of the reward-associated stimulus. Evidence for this latter possibility comes from a study by Hickey et al. (2010), who had participants search for a shape-defined target (e.g., a red diamond in an array of red circles) while one of the nontarget shapes was rendered in a unique colour (e.g., green), so as to be a coloursingleton distractor. Participants received rewards for correct responses; the size of this reward varied randomly from trial to trial. The key finding from this study was that an electrophysiological marker of early sensory processing in the extrastriate visual cortex (Hillyard, Vogel, \& Luck, 1998) was larger for a distractor rendered in a colour that had been used as a highly rewarded target on the immediately preceding trial. This finding is consistent with the idea that reward alters the perceptual salience of stimuli on a trial-by-trial basis.

However, some of the present findings do not sit so neatly with the idea of reward information biasing 
competition on the saccade map through a process of competitive integration. In particular, the competitive integration model (Godijn \& Theeuwes, 2002) predicts that increasing activity at one location on the priority map should not only have an influence on the likelihood of making a saccade in the direction of that peak but also should influence the latency of saccades. For example, if reward learning augments the saccadic activity associated with the location of a high-value distractor, this should also increase the inhibition of saccadic activity at the distant target location, such that it takes longer for the activity peak associated with the target to reach the saccade threshold (i.e., the remote distractor effect; cf. Godijn \& Theeuwes, 2002). Therefore, on trials where first saccades go towards the target, a larger remote distractor effect should be evident when the high-value distractor is present in the display than when the low-value distractor is present. However, in the current experiments, there was no effect of reward on the latency of saccades made towards either stimulus. Notably, some previous studies investigating the influence of reward on oculomotor capture have similarly found no evidence that saccades to the target are slowed more by the presence of a high-value distractor than a low-value distractor (Pearson et al., 2015, Experiment 1; Theeuwes \& Belopolsky, 2012), although others have found evidence for such an effect (Le Pelley et al., 2015; Pearson et al., 2015, Experiment 2). This raises a number of possible alternative explanations for how reward information might be incorporated into the saccade priority map. For instance, it may be that the association between the critical stimulus feature and reward gives an independent boost to the saccadic activity associated with that feature, without affecting the lateral inhibition of other stimuli in the visual field (Belopolsky, 2015). Alternatively, reward information may lower the saccade threshold in a feature-specific manner, such that less saccadic activity is required to initiate a saccade towards a specific stimulus feature that is associated with high-value reward. However, as the current data adds to a set of mixed findings within the literature, it remains for future studies to establish whether the presence of a reward-associated distractor stimulus reliably lengthens the latency of saccades to a target, and thus whether the competitive integration model can fully account for the VMOC effect.

\section{Conclusions}

The current experiments demonstrate that value-modulated oculomotor capture (VMOC) by task-irrelevant, physically salient stimuli is a consequence of early competition on the saccadic priority map. That is, learning that a stimulus feature signals large reward increases the strength of that feature's representation on the saccade map in a way that mimics or interacts with physical salience, such that stimuli possessing those features are more likely to capture attention in future.

Author Note This work was supported by a grant from the Australian Research Council (FT100100260). Jan Theeuwes was supported by an ERC advanced grant (ERC-2012-AdG-323413). We would like to thank Nick Yeung for his helpful advice during the preparation of the manuscript.

\section{References}

Anderson, B. A. (2013). A value-driven mechanism of attentional selection. Journal of Vision, 13, 7-7. doi:10.1167/13.3.7

Anderson, B. A., Laurent, P. A., \& Yantis, S. (2011a). Learned value magnifies salience-based attentional capture. PloS One, 6, e27926. doi:10.1371/journal.pone.0027926

Anderson, B. A., Laurent, P. A., \& Yantis, S. (2011b). Value-driven attentional capture. Proceedings of the National Academy of Sciences of the United States of America, 108, 10367-10371. doi:10.1073/ pnas. 1104047108

Anderson, B. A., Laurent, P. A., \& Yantis, S. (2013). Reward predictions bias attentional selection. Frontiers in Human Neuroscience, 7, 262. doi:10.3389/fnhum.2013.00262

Anderson, B. A., \& Yantis, S. (2012). Value-driven attentional and oculomotor capture during goal-directed, unconstrained viewing. Attention, Perception, \& Psychophysics, 74, 1644-1653. doi:10. 3758/s13414-012-0348-2

Awh, E., Belopolsky, A. V., \& Theeuwes, J. (2012). Top-down versus bottom-up attentional control: A failed theoretical dichotomy. Trends in Cognitive Sciences, 16, 437-443. doi:10.1016/j.tics. 2012.06.010

Beesley, T., Pearson, D., \& Le Pelley, M. (2015). Implicit learning of gaze-contingent events. Psychonomic Bulletin \& Review, 22, 800 807. doi: $10.3758 / \mathrm{s} 13423-014-0720-4$

Belopolsky, A. V. (2015). Common priority map for selection history, reward and emotion in the oculomotor system. Perception, 1-14. doi: $10.1177 / 0301006615596866$

Brainard, D. H. (1997). The psychophysics toolbox. Spatial Vision, 10, 433-436. doi:10.1163/156856897X00357

Chelazzi, L., Perlato, A., Santandrea, E., \& Della Libera, C. (2013). Rewards teach visual selective attention. Vision Research, 85, 5862. doi:10.1016/j.visres.2012.12.005

Cousineau, D. (2005). Confidence intervals in within-subject designs: A simpler solution to Loftus and Masson's method. Tutorials in Quantitative Methods for Psychology, 1, 42-45.

Della Libera, C., \& Chelazzi, L. (2009). Learning to attend and to ignore is a matter of gains and losses. Psychological Science, 20, 778-784. doi:10.1111/j.1467-9280.2009.02360.x

Donk, M., \& van Zoest, W. (2008). Effects of salience are short-lived. Psychological Science, 19, 733-739. doi:10.1111/j.1467-9280. 2008.02149.x

Failing, M., Nissens, T., Pearson, D., Le Pelley, M., \& Theeuwes, J. (2015). Oculomotor capture by stimuli that signal the availability of reward. Journal of Neurophysiology, 114, 2316-2327. doi:10. 1152/jn.00441.2015

Failing, M., \& Theeuwes, J. (2014). Exogenous visual orienting by reward. Journal of Vision, 14, 6. doi:10.1167/14.5.6

Godijn, R., \& Theeuwes, J. (2002). Programming of endogenous and exogenous saccades: Evidence for a competitive integration model. 
Journal of Experimental Psychology: Human Perception and Performance, 28, 1039-1054. doi:10.1037/0096-1523.28.5.1039

Hickey, C., Chelazzi, L., \& Theeuwes, J. (2010). Reward changes salience in human vision via the anterior cingulate. Journal of Neuroscience, 30, 11096-11103. doi:10.1523/JNEUROSCI.102610.2010

Hickey, C., Chelazzi, L., \& Theeuwes, J. (2011). Reward has a residual impact on target selection in visual search, but not on the suppression of distractors. Visual Cognition, 19, 117-128. doi:10.1080/ 13506285.2010 .503946

Hillyard, S. A., Vogel, E. K., \& Luck, S. J. (1998). Sensory gain control (amplification) as a mechanism of selective attention: Electrophysiological and neuroimaging evidence. Philosophical Transactions of the Royal Society B: Biological Sciences, 353, 1257-1270. doi:10.1098/rstb.1998.0281

Ikeda, T., \& Hikosaka, O. (2003). Reward-dependent gain and bias of visual responses in primate superior colliculus. Neuron, 39, 693 700. doi:10.1016/S0896-6273(03)00464-1

Itti, L., \& Koch, C. (2001). Computational modelling of visual attention. Nature Reviews Neuroscience, 2, 194-203. doi:10.1038/35058500

Kamin, L. J. (1968). Attention-like processes in classical conditioning. In M. R. Jones (Ed.), Miami symposium on the prediction of behavior (pp. 9-33). Miami: University of Miami Press.

Kleiner, M., Brainard, D. H., Pelli, D. G., Broussard, C., Wolf, T., \& Niehorster, D. (2007). What's new in Psychtoolbox-3? Perception, 36, S14. doi:10.1068/v070821

Le Pelley, M. E. (2004). The role of associative history in models of associative learning: A selective review and a hybrid model. The Quarterly Journal of Experimental Psychology. B, Comparative and Physiological Psychology, 57, 193-243. doi:10.1080/ 02724990344000141

Le Pelley, M. E., Mitchell, C. J., Beesley, T., George, D. N., \& Wills, A. J. (2016). Attention and associative learning in humans: An integrative review. Psychonomic Bulletin \& Review, in press

Le Pelley, M. E., Pearson, D., Griffiths, O., \& Beesley, T. (2015). When goals conflict with values: Counterproductive attentional and oculomotor capture by reward-related stimuli. Journal of Experimental Psychology: General, 144, 158-171. doi:10.1037/xge0000037

Li, Z. (2002). A saliency map in primary visual cortex. Trends in Cognitive Sciences, 6, 9-16. doi:10.1016/S1364-6613(00)01817-9

Ludwig, C. J. H., \& Gilchrist, I. D. (2002). Stimulus-driven and goaldriven control over visual selection. Journal of Experimental Psychology: Human Perception and Performance, 28, 902-912. doi:10.1037/0096-1523.28.4.902

Ludwig, C. J. H., \& Gilchrist, I. D. (2003a). Goal-driven modulation of oculomotor capture. Perception \& Psychophysics, 65, 1243-1251. doi:10.3758/BF03194849

Ludwig, C. J. H., \& Gilchrist, I. D. (2003b). Target similarity affects saccade curvature away from irrelevant onsets. Experimental Brain Research, 152, 60-69. doi:10.1007/s00221-003-1520-7

Mackintosh, N. J. (1975). A theory of attention: Variations in the associability of stimuli with reinforcement. Psychological Review, 82, 276-298. doi:10.1037/h0076778

Meeter, M., Van der Stigchel, S., \& Theeuwes, J. (2010). A competitive integration model of exogenous and endogenous eye movements. Biological Cybernetics, 102, 271-291. doi:10.1007/s00422-0100365-y

Mitchell, C. J., \& Le Pelley, M. E. (Eds.). (2010). Attention and associative learning: From brain to behaviour. Oxford: Oxford University Press.
Mulckhuyse, M., van Zoest, W., \& Theeuwes, J. (2008). Capture of the eyes by relevant and irrelevant onsets. Experimental Brain Research, 186, 225-235. doi:10.1007/s00221-007-1226-3

Pearson, D., Donkin, C., Tran, S. C., Most, S. B., \& Le Pelley, M. E. (2015). Cognitive control and counterproductive oculomotor capture by reward-related stimuli. Visual Cognition, 23, 41-66. doi: 10.1080/13506285.2014.994252

Pelli, D. G. (1997). The VideoToolbox software for visual psychophysics: Transforming numbers into movies. Spatial Vision, 10, 437-442. doi:10.1163/156856897X00366

Ratcliff, R. (1979). Group reaction time distributions and an analysis of distribution statistics. Psychological Bulletin, 86, 446-461. doi:10. 1037/0033-2909.86.3.446

Rutherford, H. J. V., O’Brien, J. L., \& Raymond, J. E. (2010). Value associations of irrelevant stimuli modify rapid visual orienting. Psychonomic Bulletin \& Review, 17, 536-542. doi:10.3758/PBR. 17.4.536

Salvucci, D. D., \& Goldberg, J. H. (2000). Identifying fixations and saccades in eye-tracking protocols. In Proceedings of the symposium on eye tracking research \& applications - ETRA '00 (pp. 71-78). New York: ACM Press.

Theeuwes, J. (1992). Perceptual selectivity for color and form. Perception \& Psychophysics, 51, 599-606. doi:10.3758/BF03211656

Theeuwes, J. (1994). Endogenous and exogenous control of visual selection. Perception, 23, 429-440. doi:10.1068/p230429

Theeuwes, J., \& Belopolsky, A. V. (2012). Reward grabs the eye: Oculomotor capture by rewarding stimuli. Vision Research, 74, 80-85. doi:10.1016/j.visres.2012.07.024

Theeuwes, J., Kramer, A. F., Hahn, S., Irwin, D. E., \& Zelinsky, G. J. (1999). Influence of attentional capture on oculomotor control. Journal of Experimental Psychology: Human Perception and Performance, 25, 1595-1608. doi:10.1037/0096-1523.25.6.1595

Thorndike, E. L. (1911). Animal intelligence: Experimental studies. New York: Macmillan.

Trabasso, T., \& Bower, G. H. (1968). Attention in learning: Theory and research. New York: Wiley.

Trappenberg, T. P., Dorris, M. C., Munoz, D. P., \& Klein, R. M. (2001). A model of saccade initiation based on the competitive integration of exogenous and endogenous signals in the superior colliculus. Journal of Cognitive Neuroscience, 13, 256-271. doi:10.1162/ 089892901564306

van Zoest, W., Donk, M., \& Theeuwes, J. (2004). The role of stimulusdriven and goal-driven control in saccadic visual selection. Journal of Experimental Psychology: Human Perception and Performance, 30, 746-759. doi:10.1037/0096-1523.30.4.749

Vokoun, C. R., Mahamed, S., \& Basso, M. A. (2011). Saccadic eye movements and the basal ganglia. In S. P. Liversedge, I. D. Gilchrist, \& S. Everling (Eds.), The Oxford handbook of eyemovements (pp. 215-234). New York: Oxford Universty Press.

Walker, R., Walker, D. G., Husain, M., \& Kennard, C. (2000). Control of voluntary and reflexive saccades. Experimental Brain Research, 130, 540-544. doi:10.1007/s002219900285

Wolfe, J. M., Cave, K. R., \& Franzel, S. L. (1989). Guided search: An alternative to the feature integration model for visual search. Journal of Experimental Psychology: Human Perception and Performance, 15, 419-433. doi:10.1037/0096-1523.15.3.419

Yantis, S. (2000). Goal-directed and stimulus-driven determinants of attentional control. In S. Monsell \& J. Driver (Eds.), Control of cognitive processes: Attention and performance XVII (pp. 73-103). Cambridge: MIT Press. 